\title{
Protección financiera en salud: México, 1992 a 2004
}

Felicia Marie Knaul, Dra en Econ, (1) Héctor Arreola-Ornelas, M en Econ S, (2) Oscar Méndez, Analista. ${ }^{(3)}$

\author{
Knaul FM,Arreola-Ornelas H, Méndez O. \\ Protección financiera en salud: México, 1992 a 2004. \\ Salud Publica Mex 2005;47:430-439
}

\section{Resumen}

Objetivo. Documentar las tendencias en la protección financiera del sistema de salud en México en el periodo de 1992 a 2004, aplicando una serie de indicadores que miden el gasto en salud catastrófico y empobrecedor y el Indice de Justicia en la Protección Financiera. Material y métodos. El estudio hace uso de la serie de tiempo bianual de la Encuesta de Ingresos y Gastos de los Hogares (ENIGH) en el periodo 1992 a 2004. La metodología aplicada busca medir el nivel y la distribución de la carga del financiamiento a la salud de los hogares, y la protección financiera que les ofrece el sistema de salud. Para ello, se presentan cuatro indicadores: I) el índice de justicia en la contribución financiera; 2) la proporción de hogares con gastos catastróficos; 3 ) la proporción de hogares con gastos en salud empobrecedores; $y$ 4) la suma entre el porcentaje de hogares con gastos catastróficos y empobrecedores, lo cual se denomina gastos excesivos. El análisis presentado en este documento es descriptivo, dejando para estudios posteriores el análisis de los aspectos causales a una mayor profundidad. Resultados. Se estima que el número de familias con gastos en salud empobrecedores aumentó desde $5.2 \%$ en 1992 hasta alcanzar su nivel más alto de $9.9 \%$ en 1996 y posteriormente declinar en forma gradual y llegar a I.8\% en 2004. En cuanto al porcentaje de hogares con gastos catastróficos, inició en 1992 en $2.8 \%$ y aumentó a $4.2 \%$ en 1998, para posteriormente presentar una mejoría continua y llegar en 2004 a un nivel de $2.6 \%$. La mejoría desde 2000 se concentra entre la población no asegurada, las familias afiliadas al Seguro Popular y los hogares de los dos quintiles más pobres. Conclusiones: Los gastos excesivos han tenido un comportamiento que refleja un empeoramiento en el periodo de crisis económica, una mejora postcrisis y una mejoría sostenida a partir del 2000. Las cifras sugieren que una parte de la reducción en el número de ho-
Knaul FM, Arreola-Ornelas H, Méndez O.

Financial protection in health: Mexico, 1992 to 2004. Salud Publica Mex 2005;47:430-439

\begin{abstract}
Objective. To document trends in financial protection in the health care system in Mexico between 1992 and 2004, applying a series of indicators that measure catastrophic and impoverishing health spending and the Index of Fairness in Financial Contributions. Materials y Methods. This study uses the biannual time series of the Survey of Household Income and Expenditures (ENIGH) spanning the period 1992 to 2004. The methodologies seek to measure the level and distribution of the burden of health care finance on households, and the financial protection offered to them by the health care system. Four indicators are presented: I) the Index of Fairness in Financial Contributions, 2) the proportion of households with catastrophic health expenditure, 3) the proportion of households with impoverishment due to health spending and 4) the sum of the proportion of households with catastrophic or impoverishing health spending, which is referred to as excessive health spending.The analysis presented in this document is descriptive, leaving for later studies a deeper analysis of causal aspects. Results. The number of families that suffer impoverishment due to health spending increased from $5.2 \%$ in 1992, reached a high of $9.9 \%$ in 1996 , and then gradually declined to $1.8 \%$ by 2004. The proportion of households with catastrophic expenses began at $2.8 \%$ in 1992, increased to $4.2 \%$ in 1998 and then fell to a level of $2.6 \%$ in 2004. The improvements from 2000 on are concentrated among the uninsured population, families affiliated to Popular Health Insurance, and households in the poorest two quintiles of the income distribution. Conclusions. The patterns over time in excessive health spending reflect a worsening during periods of economic crisis, post-crisis recovery, and a sustained improvement beginning in the year 2000. The data suggest that part of the reduction in the number of households with excessive
\end{abstract}

(I) Coordinadora General para la Modernización Administrativa de la Educación, Secretaría de Educación Pública. Economista Principal (con licencia), Fundación Mexicana para la Salud.

(2) Coordinador de Investigación en Economía de la Salud, Competitividad y Salud, Fundación Mexicana para la Salud.

(3) Investigador, Competitividad y Salud, Fundación Mexicana para la Salud.

Fecha de recibido: 29 de agosto de 2005 - Fecha de aprobado: 15 de noviembre de 2005

Solicitud de sobretiros: M. en Econ. S. Héctor Arreola-Ornelas, FUNSALUD, Periférico Sur No. 4809, colonia Arenal Tepepan, I46I0 México, D.F. Correo electrónico: harreola@funsalud.org.mx 
gares con gastos excesivos se debe a la ampliación de la protección financiera de las familias mexicanas que ha traído consigo el Seguro Popular de Salud, mientras otra parte se asocia a reducciones en la pobreza. También son indicativos de una importante relación entre el comportamiento de la economía en general y los gastos catastróficos y empobrecedores en salud, lo cual sugiere el importante rol del aseguramiento en salud para blindar a las familias. La protección financiera ayuda a garantizar que cuando los tiempos de crisis económica - del país o de una familia - coinciden con eventos de enfermedad, el gasto en la atención a la salud no se vuelve causa de un periodo largo o permanente de empobrecimiento para los hogares.

Palabras clave: protección financiera; Seguro Popular; Sistema de Protección Social en Salud; gasto del bolsillo en salud; gasto catastrófico; índice de justicia en la contribución financiera; empobrecimiento; crisis económica; sistema de salud de México; México health spending is due to the extension of financial protection for Mexican families through the Popular Health Insurance, while another part is associated with a decline in poverty. In addition, this paper documents an important relationship between economic trends and catastrophic and impoverishing health spending, suggesting the importance of financially protecting families through health insurance. Financial protection assists in guaranteeing that when economic crisis - of a country or of a family - coincides with illness, health care payments do not become the cause of a long or permanent period of impoverishment for households.

Key words: financial protection; Popular Health Insurance, System for Social Protection in Health, out-of-pocket health spending, catastrophic health spending, index of fairness in financial contributions, impoverishment, economic crisis, Mexican health system; Mexico
L a necesidad de mejorar la justicia en el financiaImiento es uno de los retos más apremiantes para el sector salud mexicano. En el marco de la Organización Mundial de la Salud (OMS) para medir el desempeño de los sistemas de salud, la posición de México sufrió por su pobre alcance en lo que respecta a justicia financiera. ${ }^{1}$ El puntaje de México lo ubicó en el lugar 51 en términos del logro general entre un total de 191 países evaluados. En justicia financiera del sistema de salud, la cual tiene un peso importante en el cálculo global, México obtuvo la posición 144.*

El bajo desempeño del sistema de salud mexicano es reflejo de la falta de protección financiera que vivía más de la mitad de la población - los hogares que carecían de acceso a un seguro público o privado en saludantes de la reforma del 2003. ${ }^{2}$ El anterior sistema de salud en México, como en varios otros países de América Latina, se basó en un modelo fragmentado en el cual las familias de bajos recursos económicos y la población excluida del sector formal tenían un menor acceso a las prestaciones en salud y carecían de oportunidades para participar en esquemas de aseguramiento. ${ }^{3}$

La falta de protección financiera se traduce no sólo en gastos en salud excesivos — catastróficos y empobrecedores - sino también en menor acceso, baja calidad en los servicios de salud e ineficiencia en el uso de los

\footnotetext{
* Cabe señalar que la medición de la protección financiera en la OMS 2000 está basada en datos de la Encuesta de Ingresos y Gastos de los Hogares (ENIGH) de 1996. En contraste, los resultados del presente estudio correspondiente al año 2000 son de la ENIGH de 2000 .
}

recursos del sector salud. Un indicador determinante de la falta de protección financiera es la proporción del gasto en salud que es solventado directamente del bolsillo de los hogares, ${ }^{4,5}$ la cual es considerada como la forma menos eficiente y menos justa de financiar un sistema de salud. ${ }^{1,6-9}$ Lo anterior provoca, a su vez, que las familias enfrenten gastos empobrecedores y catastróficos. ${ }^{10,11}$ El pago directo del bolsillo de los hogares representó 52\% del gasto total en salud en $2004 .^{10}$

Esta situación se complica aun más debido a su combinación con la falta de cobertura y acceso a la seguridad de más de la mitad de la población. Mientras que poco más de $60 \%$ de la población del quintil más rico estaba asegurada en 2000, para el quintil más pobre la cifra era de apenas $10 \%$. En total $6.3 \%$ de los hogares presentaron gastos catastróficos o empobrecedores, pero eran más comunes entre los hogares del quintil más pobre, que entre los de mayores ingresos. Además, la incidencia del gasto catastrófico y empobrecedor era más de cuatro veces mayor entre la población no asegurada. ${ }^{10-12}$

México ideó en 2003 una reforma estructural que busca dar respuesta al desafío de mejorar la justicia en el financiamiento del sistema de salud y ofrecer un programa de aseguramiento en salud para proteger a las familias más expuestas a sufrir gastos catastróficos., ${ }^{3,43-16} \mathrm{El}$ eje de la reforma es extender la protección financiera en salud a las familias no cubiertas por la seguridad social a través de un seguro universal subsidiado que se denomina el Seguro Popular de Salud (SPS). La reforma generó el Sistema de Protección Social en Salud (SPSS) con los propósitos de proteger financieramente a las familias reduciendo su gasto directo de bolsillo, proporcionar mayores incentivos para la eficiencia del sistema, promover una distribución más 
equitativa de los recursos financieros en salud y ofrecer una mejor calidad en la atención, entre otros. ${ }^{3,4,13}$

El análisis presentado por la Secretaría de Salud de México que dio sustento a la creación del Sistema de Protección Social en Salud enfatizó a la segmentación del sistema de salud y a la falta de aseguramiento para una proporción importante de la población, aunado a las transiciones epidemiológica y demográfica. Ello dio lugar a cinco grandes desequilibrios financieros: 1) con respecto al nivel de la inversión llevada a cabo; 2) referente al origen de los fondos para el financiamiento, que es mayoritariamente privado en relación al público; 3) con respecto a la distribución, la cual favoreció principalmente a las instituciones de seguridad social en detrimento de la población no asegurada; 4) en relación con la corresponsabilidad federal versus la estatal, en lo cual existe una gran diferencia entre la proporción del financiamiento proveniente de aportaciones estatales entre las diferentes entidades; y 5) con respecto al destino de los fondos, donde se documenta una cada vez mayor proporción de recursos destinada al pago de nómina en decremento del monto destinado a inversión. .,14-17 $^{-17}$

El SPS funcionó como un programa piloto de 2001 a 2003. Por su parte, la reforma fue aprobada en abril de 2003 y el SPSS empezó a operar formalmente el 1 de enero de 2004 con la meta de alcanzar la cobertura universal en salud antes de 2010. A finales de 2004 el sistema había afiliado a casi 1.6 millones de familias, de las cuales $94 \%$ pertenecían a los dos primeros deciles de ingresos, ${ }^{10}$ lo cual equivale a cerca de $13 \%$ de la población que carecía de acceso a la seguridad social. A mediados de 2005 todos los estados, incluyendo el Distrito Federal, se habían incorporado al SPSS y la afiliación seguía avanzando acorde con la meta de incorporar a 1.5 millones de familias en este año.,10,18

Con el objetivo de documentar las tendencias en la protección financiera del sistema de salud en México, el presente estudio aplica una serie de indicadores que miden el gasto catastrófico y empobrecedor en el periodo de 1992 a 2004. Este estudio hace uso de una fuente de información particularmente rica: la serie de tiempo de 1992 a 2004 de las Encuestas de Ingreso y Gasto de los Hogares (ENIGH) levantadas por el Instituto Nacional de Estadística, Geografía e Informática (INEGI) cada dos años. ${ }^{19}$ Aunque la ENIGH se ha analizado ampliamente, este estudio constituye uno de los primeros esfuerzos para generar una serie de tiempo dirigida a estudiar tendencias sobre el gasto en salud. Esta base de datos tiene como ventaja ofrecer una visión de 14 años. El periodo incluye los años de crisis económica de finales de 1994 y de 1995, y que se ve reflejada en los datos de la ENIGH de 1996, y postcrisis reflejados en las ENIGH de los años 1998 y 2000. Adicionalmente sirve para establecer medidas basales sobre los niveles de protección financiera antes del arranque del programa piloto del Seguro Popular (ENIGH 2000) y de estudiar el periodo del piloto (ENIGH 2002 y 2004) y el primer año de su aplicación en el contexto del Sistema de Protección Social en Salud (ENIGH 2004). La información presentada en el estudio es de tipo descriptiva y no incluye un análisis causal de los múltiples factores que determinan el nivel de gasto en salud de los hogares y entre la población. Queda para la agenda de investigación, en posteriores análisis, establecer los mecanismos causales a través de los cuales los factores micro y macroeconómicos generan los cambios en las tendencias.

El documento consta de tres secciones. La primera parte describe la metodología, los indicadores y las fuentes de información. La segunda sección analiza las tendencias en los indicadores en el plano global, entre la población con acceso a la seguridad social y la que carece de ella, así como por nivel de ingreso. La última sección resume las conclusiones y presenta una agenda de investigación a futuro.

\section{Material y métodos}

\section{Indicadores}

La metodología aplicada en este estudio busca medir el nivel y la distribución de la carga del financiamiento a la salud de los hogares y la protección financiera que ofrece el sistema de salud para protegerlos contra riesgos financieros.

El estudio presenta cuatro indicadores para documentar las tendencias en la protección financiera en salud entre los hogares:

i) El Indice de Justicia en la Contribución Financiera $(\mathrm{IJCF}) ;{ }^{1,8}$

ii) La proporción de hogares con gastos catastróficos, medida como el porcentaje de hogares con gastos en salud mayores a $30 \%$ del ingreso disponible (ingreso total menos gasto en comida); ${ }_{i}^{8}$

iii) La proporción de hogares con gastos en salud empobrecedores, entendida como la proporción de hogares cuyo gasto en salud los lleva a sobrepasar la línea de pobreza o que ya estando por debajo de ella los lleva a un nivel de pobreza aún más profundo; ${ }^{11,19} \mathrm{y}$

iv) La suma entre el porcentaje de hogares con gastos catastróficos y empobrecedores, lo cual se denomina gastos excesivos. 3,10

Para medir la justicia en el financiamiento de los sistemas de salud, en el 2000, la OMS estableció las bases para la medición de la protección financiera. ${ }^{1,8}$ Para ello, calculan la contribución financiera de los hogares (CFH) como la contribución total del hogar a la salud 
en función de su ingreso efectivo, es decir, descontando el gasto en subsistencia. La contribución incluye todos los pagos y aportaciones al sistema de salud atribuibles a los hogares: el pago de impuestos, las contribuciones a la seguridad social, las primas de seguros privados y los pagos directos del bolsillo. El ingreso efectivo es aproximado a partir del ingreso disponible del hogar usando el gasto total de cada hogar menos su gasto en alimentación.

La distribución de la CFH, y por lo tanto la carga financiera del sistema de salud, se incorpora en el Indice de Justicia en la Contribución Financiera (IJCF), el cual se mide de la siguiente forma:

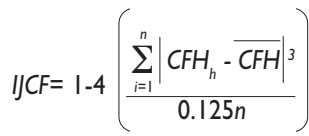

donde $h$ corresponde al $h$-ésimo hogar y $n$ al número total de hogares. ${ }^{8}$

En la ecuación 1, la diferencia absoluta entre la CFH de cada hogar y su valor medio entre todos los hogares se eleva al cubo a manera de dar un mayor énfasis a los gastos excesivos (los gastos en salud que constituyen una proporción muy elevada del ingreso del hogar). Dado que el denominador de la CFH es el ingreso disponible, el indicador asigna un peso sustancial a los hogares más pobres que tienen una mayor probabilidad de tener gastos en salud nominalmente bajos, pero que en relación con su ingreso efectivo son altos.*

El IJCF refleja una definición de la justicia financiera que busca la equidad horizontal y vertical, y la eliminación de los gastos excesivos relativos al ingreso disponible. Según esta definición: "Un sistema de salud se financia de una forma perfectamente justa, si la razón entre el gasto total per cápita en salud de cada hogar y su ingreso permanente, disponible per cápita (capacidad de pago), es idéntica para todos los hogares, independientemente del ingreso, de las necesidades o del uso del sistema de salud" ${ }^{8}$ El Indice produce una medida continua con una cota inferior en cero y superior en uno, donde los valores más cercanos a la unidad son los que indican un sistema de salud con mayor justicia financiera.

Tres indicadores complementarios, y más fácilmente interpretables, se presentan en este estudio. El primero, corresponde al gasto catastrófico en salud, lo cual se mide según la primera generación de medición de la OMS publicada en 2000.1,8 Los casos de gastos catastróficos, según

\footnotetext{
* El Indice de justicia en la protección financiera es un caso particular del estadístico diferencia individual con respecto a la media en la que $\alpha=3$ y $\beta=1$. Para una mejor referencia remitirse a Murray y colaboradores, $2000 .^{9}$
}

esta metodología, se presentan cuando la CFH es mayor a $30 \%$, y por su construcción el gasto catastrófico es una medida de gasto en salud como proporción del ingreso disponible que enfatiza los aspectos relativos de los gastos excesivos en salud. Incluye casos de gastos nominalmente bajos, pero altos como proporción del ingreso disponible de los hogares y que muchas veces implican una catástrofe financiera para la familia. A la vez incluye gastos nominalmente altos entre familias ricas que se consideran excesivos, aunque no llevan al hogar a ser pobre. El establecimiento del nivel de $30 \%$ como el punto catastrófico es subjetivo y constituye un tema para futuros análisis.

Una opción alternativa al indicador de gastos catastróficos, propuesta por Wagstaff y Van Doorslaer, es el indicador del empobrecimiento absoluto o gasto empobrecedor. ${ }^{19}$ En este estudio, se mide la proporción de hogares con gasto empobrecedor como el porcentaje de hogares que debido a su gasto en salud caen por debajo de la línea de pobreza, o bien cuando ya estando por debajo de ella los lleva a profundizar su pobreza. Para los fines del presente análisis se establece la línea de pobreza como 1 dólar por persona al día.

Definir "profundizar" constituye un gran desafío empírico y conceptual; por ello, para los fines de este estudio se emplea una aproximación basada en los datos de la Encuesta Nacional de Aseguramiento y Gasto en Salud, 2001 (ENAGS). En esta encuesta se les preguntó a las familias si debido a su gasto en salud tuvieron que modificar su gasto en alimentación, educación o vivienda, lo cual sucedió en un nivel medio del gasto en salud igual al 6-7\% del ingreso disponible. ${ }^{11}$ Dicho nivel fue elegido como el punto de corte a partir del cual se considera que un hogar profundiza su pobreza, una vez que ya ha rebasado la línea de pobreza. Nuevamente, la definición del punto de corte es tema para futuros estudios. ${ }^{11}$

Por último, la sumatoria de los indicadores de gasto catastrófico y empobrecedor - absoluto y relativo- genera un indicador compuesto de los gastos en salud que se denominan excesivos en seguimiento a la nomenclatura propuesta por la Secretaría de Salud. ${ }^{10, *}$ El resultado es menor que la suma de las partes porque hay familias cuyo gasto es emprobreceder y catastrófico. A la vez, hay familias que experimentan un gasto catastrófico y que no se empobrecen y familias con un gasto empobrecedor

\footnotetext{
* En algunos análisis posteriores a la publicación del World Health Report 2000 de la OMS, se han calculado los gastos catastróficos con un denominador distinto que hace uso de la línea de pobreza absoluta en vez del gasto en alimentación. Este estudio se sigue con la metodología original para poder hacer uso del ordenamiento mundial de la OMS publicado en 2000 y para generar una serie de tiempo comparable. Para mayor detalle ver Xu et al., 2003, y Sesma et al., 2005. ${ }^{21,22}$
} 
que no rebasan 30\% del ingreso disponible. Sumando las categorías, se garantiza la inclusión de familias con muy alto, y quizá injusto, gasto nominal, aunque éste no las lleve a empobrecerse y a la vez la de familias que experimentan un gasto en salud nominalmente bajo pero que implica pasar la línea de pobreza absoluta. Estas categorías se resumen en la figura 1.

El análisis incluye una estimación del número de "casos evitados" de hogares con gastos excesivos. Este cálculo se basa en determinar a cuánto hubiese ascendido el número de hogares con dicho tipo de gastos y sin aseguramiento, de haberse mantenido el porcentaje estimado para el año 2000 entre la población no asegurada (correspondiente a 9.6\%). ${ }^{23}$

Los resultados se presentan por nivel de aseguramiento en salud y quintil de gasto per cápita. La construcción de los quintiles de ingreso es a partir del gasto per cápita y no propiamente del ingreso, porque el gasto proporciona una mejor aproximación del ingreso permanente del hogar.

\section{Fuentes de información}

La base de datos empleada para el análisis es la serie de tiempo de las ENIGH de 1992 a 2004 levantadas por el INEGI cada dos años. ${ }^{*}$ Es una encuesta de corte transversal que comprende una muestra representativa en los ámbitos nacional y subnacional (por estrato o lugar de residencia). Se acopian datos detallados sobre todas las fuentes de ingresos y gastos de cada familia. Se reúne información individual sobre la afiliación a la seguridad social, la participación en el mercado laboral, los salarios, la educación y las características sociodemográficas básicas. Se levantan entre agosto y noviembre y los datos sobre el gasto en salud se basan en los gastos incurridos durante los tres meses previos al levantamiento de la encuesta. El tamaño de la muestra de hogares de cada una de las encuestas se presenta en el cuadro I. El cuestionario base y la cobertura han sido muy estables a partir de 1992, lo cual permite una gran comparabilidad entre las encuestas en cada momento del tiempo. ${ }^{\ddagger}$

\footnotetext{
* Las ENIGH comenzaron antes de 1992; sin embargo, no es sino hasta ese año que empiezan a tener una periodicidad bianual y un cuestionario más estable.

‡ Cabe señalar que el presente estudio no requirió de la aprobación de ningún comité de ética debido a que no se realiza ningún tipo de experimentación con individuos. La información que se emplea se basa en una encuesta autorizada por la Ley de Información Estadística y Geográfica en la que el INEGI mantiene como compromiso la confidencialidad de la información y requiere del consentimiento de los hogares para su aplicación.
}

Para poder aplicar los cálculos de los indicadores sobre la serie de tiempo, en cada uno de los años disponibles de la ENIGH, se generó una base de datos con información sobre los gastos que realizan los hogares (salud, gasto total, alimentos, etc.), así como sus ingresos y demás datos relacionados con el hogar. De forma paralela, se estimaron los pagos que efectuarían los hogares por concepto de impuestos y pagos a la seguridad social basados en sus gastos, ingresos y tipo de aseguramiento, este último con base en la Ley del Instituto Mexicano del Seguro Social vigente y del Instituto de Seguridad y Servicios Sociales para los Trabajadores del Estado en cada año. Se definió como hogar no asegurado a aquél en donde ningún miembro declara el derecho a la cobertura a ningún organismo de seguro, público o privado. Se partió del supuesto, además, de que un pago cubre a una familia entera. Posteriormente, se llevó a cabo una homogenización de todas las variables en las que los códigos fueran diferentes por las modificaciones en los cuestionarios, a través de los años.

A fin de diferenciar los ciclos económicos y estructurales en el periodo de análisis, la serie de tiempo se dividió en cuatro momentos denominados como: 1) pe-

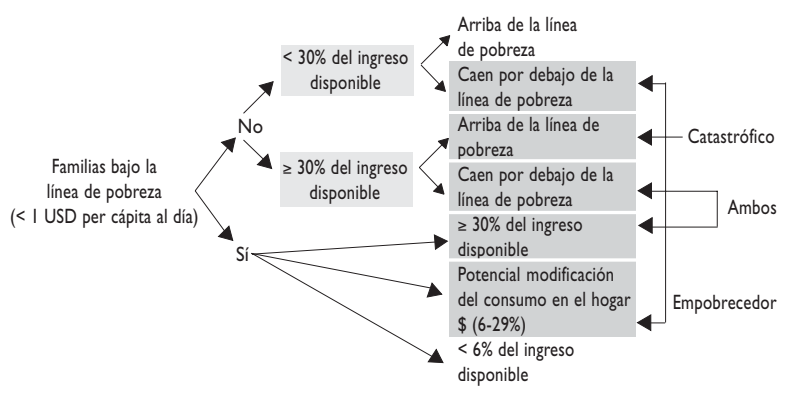

Figura I. EMPobrecimiento por gastos en salud CATASTRÓFICOS Y EMPOBRECEDORES

\section{Cuadro I \\ ENCUESTAS DE INGRESOS Y GASTOS De los hogares (ENIGH) en México}

\begin{tabular}{cc} 
Año & Hogares en muestra \\
1992 & 10503 \\
\hline 1994 & 1281 \\
\hline 1996 & 14042 \\
\hline 1998 & 10952 \\
\hline 2000 & 10108 \\
\hline 2002 & 17167 \\
\hline 2004 & 22595
\end{tabular}


riodo anterior a la crisis económica, la cual se podrá apreciar en las ENIGH de 1992 a 1994 (la ENIGH de 1994 fue aplicada antes de la crisis); 2) periodo de crisis económica que va de finales del 1994 a 1996 y que se mide con los datos de las ENIGH 1996 y 1998; 3) postcrisis y periodo de recuperación reflejados en las ENIGH de 1998 y 2000; y 4) implantación del Seguro Popular y del SPSS abarcando desde el arranque de las pruebas piloto en cinco estados (Aguascalientes, Jalisco, Tabasco, Campeche y Oaxaca) en 2001 y su primer año de operación de 2002 hasta 2004, y por ende las dos últimas aplicaciones de la ENIGH.

Es importante señalar que el análisis de los datos de 1992 a 2000 hace una diferenciación sencilla entre la población asegurada (afiliada al IMSS u otro aseguramiento público o privado) y la población no asegurada (antes llamada "abierta"). En el análisis referente a los datos posteriores a 2000 se hace referencia a dos grupos: la población sin aseguramiento o con Seguro Popular y la población asegurada con el IMSS o cualquier otro seguro de salud que no sea el Seguro Popular. Esta diferenciación, basada en los datos de la ENIGH, no supone poder identificar exactamente cuáles hogares estén afiliados al Seguro Popular; simplemente considera que a través del tiempo una parte creciente de la población que carece de otras formas de aseguramiento (IMSS, ISSSTE, PEMEX, privado, etc), se ha ido afiliando al Seguro Popular. ${ }^{14}$

Para simplificar su presentación, este documento incluye únicamente los porcentajes sencillos; sin embargo, fueron estimados los intervalos de confianza al $95 \%$ mediante la técnica de bootstraping, para cada uno de los indicadores mencionados. Estas estimaciones están disponibles a través de los autores.

\section{Resultados}

Los gastos excesivos han tenido un comportamiento que refleja un empeoramiento en el periodo de crisis económica, una mejoría en la postcrisis y una mejora sostenida a partir de 2000 (figura 2). En general, la recuperación sobrepasó la crisis y desde 1992 el porcentaje de hogares con gastos catastróficos o empobrecedores ha bajado de $7 \%$ a $4.1 \%$. A la vez, el Indice de Justicia en la Protección Financiera ha mejorado de 0.921 a 0.926.

El comportamiento entre las variables, si bien es consistente, muestra ciertas diferencias a través del tiempo. En primer lugar, los cambios en el porcentaje de hogares con gastos empobrecedores han sido más notorios. El porcentaje de familias aumento de $5.2 \%$ en 1992 y 5.5\% en 1994 a su nivel más alto de $9.9 \%$ inmediatamente des- pués de la crisis en 1996. De ahí muestra un decline gradual y llega a 7.4\% en 1998, 3.8\% en 2000, 2.4\% en 2002 y $1.8 \%$ en 2004 . En cuanto al porcentaje de hogares con gastos catastróficos, el comportamiento muestra una variación menos amplia. Se nota un aumento de 3.4\% en 1996 a $4.2 \%$ en 1998, lo cual es la fase postcrisis. Durante el periodo 2000 a 2004 hubo una mejoría continua de 3.4\% a $2.8 \%$ en 2002 y $2.6 \%$ en 2004 . Los cambios en la proporción de hogares tanto con gastos catastróficos como empobrecedores y el IJCF son significativos a partir de 1998 y hasta 2004, presentando una tendencia a la mejora en cada uno de ellos a partir de ese momento. Los intervalos de confianza para cada uno de estos indicadores (excepto para el porcentaje de hogares con gastos catastróficos entre 2002 y 2004) no presentan traslape año con año.

Por otro lado, el IJCF empeoró entre 1992 y 1994, mejoró un poco en 1996 y empeoró considerablemente entre 1996 y 1998. Esta última cifra resulta difícil de explicar y requiere de mayor estudio. Desde 2000, y después del deterioro de mediados de los años noventa, hay una mejoría continua y nuevamente esta variable llega en 2004 a su nivel más alto en todo el periodo estudiado. Este importante aumento en el nivel de protección financiera reflejado en el IJCF puede ser considerado en el contexto del lugar que obtuvo México en el marco de la OMS en 2000 con base en el IJCF de la ENIGH de 1996. El nivel de 0.926 alcanzado en 2004 es equivalente a una ganancia de 37 posiciones en el ran-

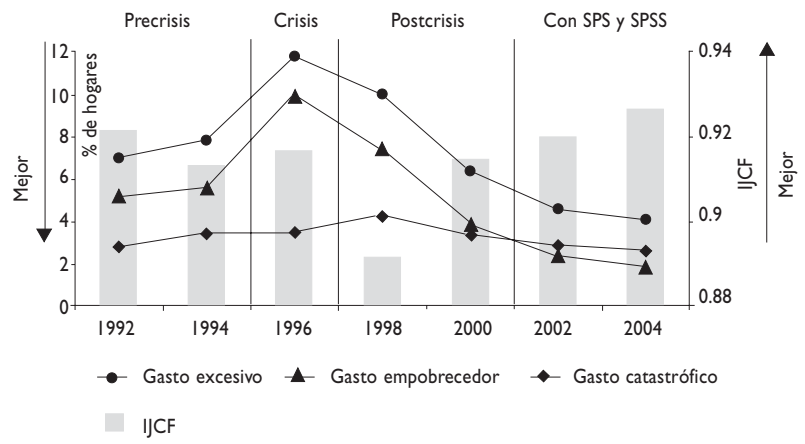

Fuente: Estimaciones propias Competitividad y Salud, FUNSALUD, con base en datos de la ENIGH

Figura 2. Tendencias de los gastos en salud de los HOGARES (1992-2004) 
king que en materia de protección financiera construyó la OMS para 191 países en el año 2000.*

El deterioro en la protección financiera, medido en términos de los gastos emprobrecedores y catastróficos de los hogares, afectó tanto a las familias con acceso a la seguridad social como a las no aseguradas (figura 3). Sin embargo, desde 2000 la mejoría se muestra únicamente entre la población no asegurada o con Seguro Popular. Para esta población, hay una reducción de $9.6 \%$ en 2000 a $6.5 \%$ en 2002 y $5.3 \%$ en 2004 . Entre los hogares con seguridad social, las cifras son $2.2 \%, 1.9 \%$ y $2.5 \%$, respectivamente. Todos los cambios en gasto excesivo entre la población no asegurada a partir de 1994 son significativos (no hay traslape en los intervalos de confianza). Para la población asegurada y los gastos catastróficos en general, los cambios tienden a no ser significativos.

Por otro lado, la mejoría en la incidencia de los gastos excesivos se concentra entre los hogares de los dos quintiles más pobres y es en estos grupos donde se ha concentrado el Seguro Popular (en 2004, más de 90\% de las familias afiliadas al Seguro Popular pertenecían al $20 \%$ más pobre de la población ${ }^{18}$ ) (figura 4). ${ }^{-}$Entre los hogares del quintil más pobre de la población, el porcentaje con gastos catastróficos o empobrecedores era de $19.6 \%$ en $2000,12.6 \%$ en 2002 y $9.7 \%$ en 2004 . En el segundo quintil, las cifras eran $4.2 \%, 3.0 \%$ y $2.5 \%$, respectivamente. Estos cambios son significativos, pues no hay traslape en los intervalos de confianza. Para la población más rica, no hay una tendencia clara.
La mejora en la protección financiera representa una reducción importante en el número de familias que experimentan gastos excesivos para financiar su salud. Las cifras de la ENIGH sólo informan respecto al trimestre anterior al levantamiento de la encuesta. En 2000, 1.48 millones de familias reportaron gastos catastróficos o empobrecedores en el trimestre anterior a la encuesta y esta cifra se reduce a 1.13 millones en 2002 y 1.05 millones en 2004, considerando incluso el crecimiento poblacional y el número de hogares. El número de eventos que se hubieran presentado si el porcentaje de familias con gastos excesivos registrado en 2000 se aplica a la población en 2004, tomando en cuenta únicamente la población no asegurada en 2000 y no asegurada o con Seguro Popular en 2004, la reducción de $9.6 \%$ a $5.3 \%$ de los hogares con gastos excesivos equivale a evitar 630 mil eventos catastróficos o empobrecedores por trimestre.

Los resultados acerca de las tendencias en la distribución entre los hogares que sufren gastos catastróficos versus gastos empobrecedores demuestran que junto con la reducción en el número total se ha presentado un cambio importante en la composición de los gastos excesivos. Desde el periodo de la crisis económica ha habido una mejoría en cada uno de las cinco categorías (descritas en la figura 1) de gasto empobrecedor y catastrófico. Sin embargo, tanto el aumento durante la crisis como la reducción posterior a ella se concentran entre las familias debajo de la línea de pobreza, incluyendo las que tienen gasto catastrófico (mayor a $30 \%$ ) y las que no (gastan menos que 30\%). El
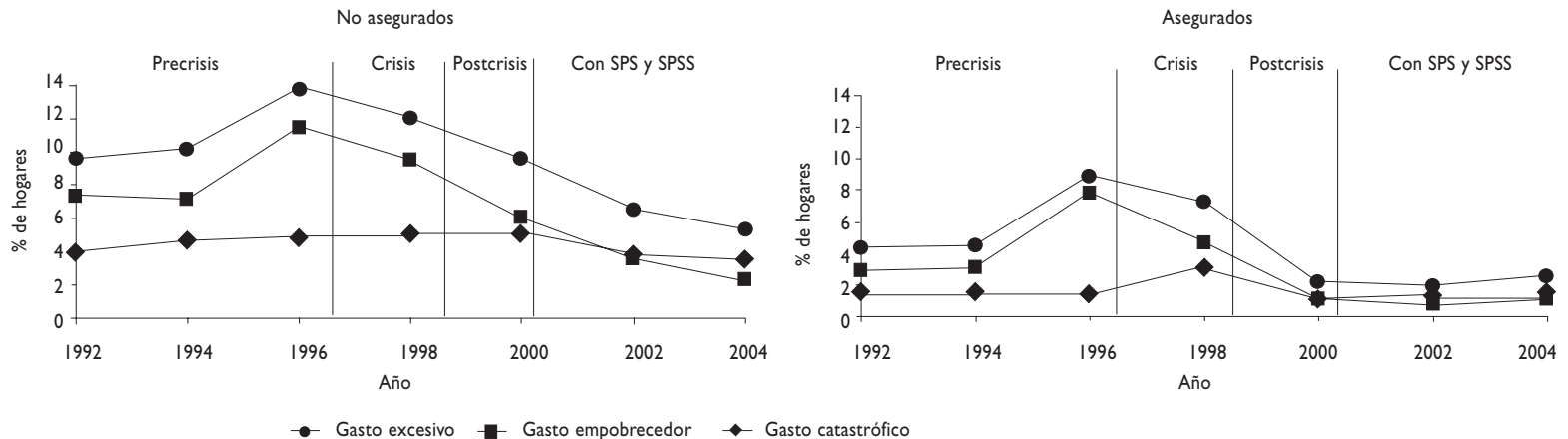

Fuente: Estimaciones propias Competitividad y Salud, FUNSALUD, con base en datos de la ENIGH

Figura 3.Tendencias del gasto en salud de los hogares por aseguramiento (1992-2004)

\footnotetext{
* La cifra para México está basada en los datos de la ENIGH de 1996, pero varía la fecha de recopilación de los datos de los demás países considerados en el ranking. El dato presentado hace referencia al nivel de México en 2004, pero comparado con el ranking de todos los demás países publicado por la OMS en 2000. En otras palabras, es el nivel de México en 2004 si los demás países siguen en la misma posición notificada por la OMS en 2000.

$\ddagger$ Los quintiles se construyen a partir del gasto per cápita por ser mejor medida del ingreso permanente de los hogares. Sin embargo, los datos fueron replicados haciendo la construcción de los quintiles de ingreso per cápita y los resultados fueron similares.
} 


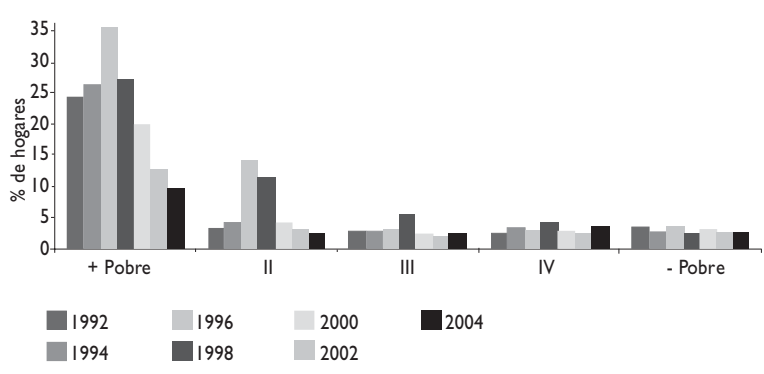

Fuente: Estimaciones propias Competitividad y Salud, FUNSALUD, con base en datos de la ENIGH

Figura 4. Evolución en LA distribución de LOS GASTOS EXCESIVOS DE LOS HOGARES DEBIDO AL GASTO EN SALUD POR QUINTIL DE INGRESOS (2000-2004)

grupo de hogares que cruza la línea de pobreza es menos grande en sí, pero entre ellos también ha habido una reducción importante desde el periodo de la crisis económica. Es entre el grupo de hogares arriba de la línea de pobreza y con gastos catastróficos (mayor a 30\%) donde la reducción ha sido menos notoria.

La reducción proporcionalmente más grande entre las familias debajo de la línea de pobreza es lo que ha generado una reversión en la composición del total de familias con gastos excesivos. Si bien antes y durante el periodo de crisis económica la mayoría de los hogares con gastos excesivos eran los que se ubicaban por debajo de la línea de pobreza y en particular los que gastaban menos que el $30 \%$ del ingreso disponible, en 2004 son los hogares arriba de la línea de pobreza con gastos catastróficos los que predominan en la distribución. Este grupo ha aumentado de $26 \%$ a $56 \%$ del total, mientras el grupo debajo de la línea de pobreza se ha reducido de $59 \%$ a $35 \%$. Las tendencias son similares entre asegurados y no asegurados.

\section{Discusión}

Este estudio documenta la evolución de los gastos catastróficos y empobrecedores en salud entre 1992 y 2004 en México. El trabajo se basa en datos de las ENIGH y por ende en una serie de tiempo larga, regular y comparable que permite observar el comportamiento del gasto en salud. Existen pocos ejemplos en el ámbito mundial de series de información sobre la protección financiera en salud de este calibre.

Los resultados muestran una tendencia de mejora en los gastos catastróficos y empobrecedores y en la justicia del financiamiento de la salud desde 1992. Dicha mejora, a lo largo del periodo, se compone de dos partes: un deterioro importante alrededor de la crisis económica que empezó a finales de 1994, y una recuperación sostenida desde esa época que culmina con mejores tasas que las registradas antes de la crisis. La mejoría después de 1998 se divide también en dos partes. La primera coincide con los años posteriores a la crisis económica. La segunda, después de la recuperación postcrisis, coincide con la implementación del Seguro Popular, y la consiguiente protección financiera que éste ofrece a las familias mexicanas contra los riesgos de empobrecerse por sus gastos en salud.

Estos resultados podrán explicarse, y son consistentes, con la hipótesis de que una parte de la reducción en el número de hogares con gastos excesivos se debe a la ampliación de la protección financiera de las familias mexicanas que ha traído consigo el Seguro Popular de Salud. Las tendencias en los gastos catastróficos y empobrecedores también coinciden con una reducción en la proporción de la población viviendo en pobreza. Desde 1998 se evidencian una reducción continua en el porcentaje de hogares que viven con menos de dos dólares al día, así como en el número de hogares que están en pobreza alimenticia (carecen del ingreso suficiente para poder adquirir una canasta básica de alimentos). ${ }^{23}$

No es posible distinguir, con los datos disponibles, entre estos efectos, especialmente por la endogenidad entre el ingreso y la salud. El cápital humano que incluye la salud es un factor clave para aumentar la productividad laboral de las personas y apoyar el proceso de salir de la pobreza. Otro componente es que existen programas complementarios al Seguro Popular, como Oportunidades, que han impactado simultáneamente en la reducción de la pobreza. ${ }^{23}$

Aunque el análisis presentado en este documento no alcanza estudiar los aspectos causales de la relación entre la expansión del Seguro Popular y la reducción del número de hogares con gastos excesivos, existen una serie de hechos relacionados con la mejoría entre 2000 y 2004 que indican un rol independiente del mejoramiento en la protección financiera del sistema de salud. En primer lugar, las mayores reducciones en los gastos excesivos entre 2000 y 2004 se observaron entre los grupos de población más pobre y entre la población no asegurada o afiliada al SPS. Los datos indican reducciones de $51 \%$ y de $41 \%$ para el primer y segundo quintil, respectivamente, y ningún cambio para los demás quintiles. Por otro lado, en términos de los hogares que en 2000 eran clasificados como no asegurados y que para 2004 pudieron continuar en esa condición o bien estar afiliados al SPS, hubo una reducción en el periodo en el porcentaje de gastos excesivos en salud de $45 \%$, mientras que entre los hogares con seguridad social ocurrió un ligero aumento. Finalmente, el hecho de que hubo una mejoría en cada una de las cinco categorías de 
gasto excesivo, incluyendo las familias que cruzan la línea de pobreza por gasto en salud, es indicativo de un efecto adicional a la reducción de la pobreza.

Será importante en futuros estudios diferenciar entre la recuperación de la economía, la reducción de la pobreza entre los hogares, otros programas y proyectos sociales y la aplicación del Seguro Popular, en términos del efecto en el gasto en salud de bolsillo de los hogares. Para poder diferenciar los factores determinantes de la reducción de los gastos excesivos, serán particularmente pertinentes el estudio a detalle de los cambios en las características de los hogares con y sin Seguro Popular en 2002 y 2004, antes y después de la crisis, así como las diferencias en las tendencias entre los estados. En futuros estudios, estas relaciones serán analizadas con técnicas econométricas y con datos de pseudopanel, los cuales darán mayor información acerca de los factores determinantes de la reducción en el porcentaje de hogares con gastos catastróficos y empobrecedores. Sin embargo, es importante señalar que para comprobar con mayor eficacia los determinantes de los cambios en la protección financiera, el estudio de datos longitudinales es esencial y ello sugiere la pertinencia de proponer la recopilación de datos de esta naturaleza para enriquecer la evaluación del impacto de las políticas del sistema de salud en la protección financiera.

La evolución de los gastos excesivos durante el periodo de 1992 a 2004 constituye evidencia importante de que el desempeño del sistema de salud, en cuanto a protección financiera, es sensible al desempeño de la economía en general. En periodos de crisis es probable que una proporción importante de familias pierda su empleo. Para los trabajos en el sector formal esto implica también la perdida del acceso a la seguridad social y así la protección financiera en salud. Por otro lado, aumenta el número de familias viviendo más cerca de la línea de pobreza y con menos oportunidades para trabajar. Estos factores reducen la capacidad de las familias para poder sostener gastos de bolsillo en salud.

Es importante tomar en cuenta que el período de crisis económica de los noventa es anterior a la puesta en marcha del Seguro Popular, lo cual implica que las familias no aseguradas y las que perdieron el acceso a la seguridad social no gozaban de otra fuente de protección financiera en salud. Es de esperarse que el Seguro Popular funcionará como un blindaje ante los periodos de crisis económica - tanto en el ámbito del país como en el de la capacidad económica de las familias-. En otras palabras, el Seguro Popular tiene la función de proteger a las familias no solamente contra los incidentes de altos gastos en salud, sino también contra los momentos de reducción temporal en su capacidad financiera general, los cuales ponen en riesgo su capa- cidad de financiar la salud. La protección financiera en salud ayuda a garantizar que cuando los tiempos de crisis económica coinciden con eventos de enfermedad, el gasto en la atención a la salud no se vuelve causa de un periodo largo o permanente de empobrecimiento.

\section{Agradecimientos}

Agradecemos el apoyo financiero e institucional del Consejo Promotor de Competitividad y Salud de la Fundación Mexicana para la Salud, del CONACyT (Ref. 38391-D) y de la Organización Mundial de la Salud (a través del National Institute on Aging, National Institutes of Health, Office of Global Health Affairs, Dept. of Health and Human Services, Grant Y1-AG-9421 OPHS9-062 Cross-country Differences in Health Systems: Economic Outcomes and Medical Care Utilization), así como los comentarios recibidos en seminarios realizados en el Instituto Nacional de Salud Pública y con el Ellison Institute de la Universidad Harvard en Cuernavaca, Morelos, y de Julio Frenk, Miguel Angel Lezana, Octavio Gómez, Paul Gertler y de los revisores. Parte del análisis metodológico fue desarrollado en un informe para la publicación titulada "Beyond Survival: Protecting households against the impoverishing effects of health shocks" que está siendo preparada por la Oficina Regional para América Latina y el Caribe del Banco Mundial. Agradecemos también al INEGI por facilitar las encuestas analizadas en este trabajo y a Fabrizio Almazán, Efrén Motta y Sonia Ortega por su apoyo en la investigación y el documento. Lo expresado en este documento es responsabilidad de los autores y no necesariamente refleja las opiniones de las instituciones que apoyaron en su preparación.

\section{Referencias}

I.World Health Organization. The World Health Report 2000. Health Systems: Improving Performance. Geneva:WHO, 2000.

2. Secretaría de Salud. Salud: México 200I. Información para la rendición de cuentas. México: SSA, 2002.

3. Knaul F, Frenk J. Health Insurance in Mexico: Achieving universal coverage through structural reform. Health Affairs 2005:2(4-6): 1467-76. 4. Frenk J, Knaul F, Goméz-Dantés $O$ et al. Financiamiento justo y protección social universal: La reforma estructural del sistema de salud en México.Working Paper prepared for the International Conference, Innovations in Health Financing (Mexico City 20-2I April 2004). México: SSA, 2004.

5. Pérez-Rico R, Sesma-Vázquez S y Puentes-Rosas E. Gasto catastrófico por motivos de salud en México: Estudio comparativo por grado de marginación. Salud Pública Mex 2005;47,Supl I:s47-s53. 6. Frenk J, Lozano R, González Block MA et al. Economía y salud: Propuesta para el avance del sistema de salud en México. Informe Final. México, D.F.: FUNSALUD, 1994. 
7. Hesketh T, Zhu WX. Health in China:The health care market. BMJ 1997:314(1616).

8. Murray CJL, Knaul F, Xu K, Mugrove P, Kawabata K. Defining and measuring fairness of financial contribution. WHO. Global Programme on Evidence Working Paper, no. 24. Ginebra:WHO, 2000.

9. Barraza-Llorens M, Bertozzi S, González-Pier E et al.Addressing inequity in health and health care in Mexico. Health Affairs 2002;2I(3):47-56

10. Secretaría de Salud. Salud: México 2004. Información para la rendición de cuentas. México: SSA, 2005.

I I. Arreola $\mathrm{H}$, Knaul F, Méndez $\mathrm{O}$ et al. Catastrophic and impoverishing health expenditure: Increasing risk pooling in the Mexican health system. Report presented to the World Bank as part of the regional study: Risk pooling, ahorro y prevención: Estudio regional de políticas institucionales para la protección de los más pobres de los efectos de los shocks de salud. México, D.F., Mimeo, 2004.

12. Knaul F, Arreola H, Borja C, Méndez O,Torres AC. El Sistema de Protección Social en Salud de México: Efectos potenciales sobre la justicia financiera y los gastos catastróficos de los hogares. En: Caleidoscopio de la salud. Knaul F y Nigenda G, ed. México, D.F.: Fundación Méxicana para la Salud, 2003.

13. Secretaría de Salud. Sistema de Protección en Salud: Hacia un sistema universal de salud. Firma del Decreto por el que se reforma y adiciona la Ley General de Salud. Diario Oficial de la Federación, I5 de Mayo de 2003. SSA. México. 2003.

14. Salud Pública de México. Siete perspectivas del Seguro Popular. Salud Pública Mex 2004; 46: 585-596.

15. Salud Pública de México. Siete perspectivas del Seguro Popular. Salud Pública Mex 2005;47: 78-89.
16. Salud Pública de México. Siete perspectivas del Seguro Popular. Salud Pública Mex 2005; 47: 166-170

17. Secretaría de Salud. Programa Nacional de Salud 200I-2006: La Democratización de la Salud en México. Hacia un sistema universal de salud. México: SSA, 2001

18. Comisión Nacional de Protección Social en Salud, Informe de resultados. Segundo semestre de 2004. Secretaría de Salud, México, D.F., 2005. [Disponible en: www.salud.gob.mx/unidades/dgpfs/files/ informesegundosemestre2004.pdf].

19. Instituto Nacional de Estadística Geografía e Informática. Encuesta Nacional de Ingresos y Gastos de los Hogares, 1992, 1994, 1996, 1998, 2000, 2002 y 2004. Aguascalientes: INEGI, México.

20. Wagstaff A, Van Doorslaer E. Catastrophe and Impoverishment in Paying for Health Care: with applications to Vietnam 1993-98. Health Economics 2003; 12: 921 -934.

21. Sesma-Vázquez S, Pérez-Rico R, Sosa-Manzano CL y Gómez-Dantés O. Gasto catastrófico por motivos de salud en México: Magnitud, distribución y determinates. Salud Pública Mex 2005; 47, Supl I: s37-s46. 22. Murray CJL, Xu K, Klavus J, Kawabata K, Hanvoravongchai P, Zeramdini $R$ et al.Assesing the distribution of household financial contributions to the health system: Concepts and empirical application. En: Murray CJL and Evans DB ed. Health systems performance assessment. Debates, methods and empiricism. Geneva:WHO, 2003: 5|3-53|.

23. Banco Mundial. Generación de Ingreso y Protección Social para los Pobres. Informe Ejecutivo. Washington: Banco Internacional de Reconstrucción y Fomento/Banco Mundial. 2005. 